\title{
Incremental Deep-Learning for Continuous Load Prediction in Energy Management Systems
}

\author{
Gustavo Aragón* \\ Harsh Puri* \\ Alexander Grass* \\ *Fraunhofer FIT \\ Sankt Augustin, Germany \\ \{name.lastname\}@fit.fraunhofer.de
}

\author{
Sisay Chala* \\ Christian Beecks ${ }^{\#, *}$ \\ "University of Münster, Germany \\ christian.beecks@uni-muenster.de
}

\begin{abstract}
In this work, we introduce load prediction as continuous input for optimization models within an optimization framework for short-term control of complex energy systems. In this context, we investigated long short-term memory (LSTM) models for load prediction, because they allow incremental training in an application with continuous real-time data and have not been used in other works for continuous load prediction to our knowledge. The test and evaluation were realized using data sets of real residential data from different locations in different time resolution - hourly and minutely. Accordingly, we tested different recurrent neural network (RNN) parameters of the model such as the number of layers, the number of hidden nodes, the inclusion of regularization, and dropout in order to find the optimal LSTM configuration for our continuous load prediction application. Besides, we analyzed the quality of the LSTM algorithm by comparing it in continuous mode with the baseline model and in batch mode with the statistical model ARIMA. Training and prediction time, as well as the error stabilization time were parameters used for the evaluation. The results showed that LSTM algorithms are highly promising for integrating continuous load prediction with incremental learning.
\end{abstract}

Index Terms-- Predictive models, load forecasting, optimization methods, energy management

\section{INTRODUCTION}

Climate change has set new challenges to the electricity sector. Nowadays, there are efforts worldwide to reduce greenhouse gas emissions. As an example, Germany plans to reduce the emissions to $55 \%$ by year 2030 compared to 1990 [1]. Energy generation plays a major role in achieving these goals. Energy generation with coal, gas and nuclear energy have emission rate factors of $960 \mathrm{gCO} 2 \mathrm{e} / \mathrm{KWh}, 460$ $\mathrm{gCO} 2 \mathrm{e} / \mathrm{KWh} 66 \mathrm{gCO} 2 \mathrm{e} / \mathrm{KWh}$, respectively [2], which underlines the toxicity of fossil fuel generation. In the case of Germany, $15,5 \%$ of energy generation was from hard coal, $28,6 \%$ from brown coal, $17,5 \%$ from nuclear energy and 13,4 $\%$ from gas in year 2017 [3]. Consequently, greenhouse gas emissions due to actual energy generation are enormous and cleaner generation with renewables is becoming essential.
However, the fluctuating character of renewable energy generation due to weather variations brings challenges for which the electrical grid is not prepared. Fluctuations can cause grid instabilities and harm the grid and the grid elements. Centralized generation techniques cannot cope with these dynamics and, therefore, new decentralized energy management techniques are required. Here, new technologies like Energy Storage Systems (ESS) combined with renewable energy generation appear as a feasible solution. Consequently, adaptive and reliable energy management systems (EMS) are required, which can integrate specific user requirements such as household owners or Distribution System Operators (DSOs). In this context, EMS provide short-term control of ESS and renewable generation management by calculating the optimal control action for the power flow in the next time step. The open-source optimization framework (OFW), that we presented in a previous work [4] uses optimization models as basis for the energy management and links predictions to these models. However, prediction uncertainties can affect the performance of the optimization depending on how effective and accurate they are. For this reason, increasing the accuracy of the predictions will improve the working of an EMS.

In this paper, we develop and implement a continuous residential load prediction. To the best of our knowledge, there is no previous work that applies predictive algorithms for continuous load predictions based on incremental learning. All approaches found in literature focus on batch data. In order to apply continuous prediction, we evaluate and compare several predictive algorithms on real residential consumption data. Furthermore, the adaptiveness of the predictions for different use cases is being demonstrated. Here, use cases are defined as load predictions for different households, where the algorithm learns stand-alone and automatically new patterns from realtime data and not in a batch form. Moreover, the algorithm is tested with data of different resolution in order to demonstrate its flexibility. The continuous load prediction will be integrated into our optimization framework [4] to enable continuous optimal control of EMS.

Traditional load prediction methods use statistical models. Autoregressive (AR), Moving Average (MA), Autoregressive 
Integrated Moving Average (ARIMA), Generalized Autoregressive Conditional Heteroscedasticity (GARCH) are examples of them [5][6]. As an example, Cao et al. (2015) used ARIMA model and a similar day method for achieving load forecasting [7]. However, these algorithms do not perform accurately with non-stationary data such as load prediction, as we will verify in Section IV. Results.

Another approach for load prediction are data-driven models. These models correspond to artificial intelligence algorithms that perform better with non-linear data. Feedforward Multilayer Perceptron (MLP), Support Vector Regression (SVR), LSTM, among others, are included in this group [8]. In [9], Tang and Zhang (2011) combines ANNs with the grey optimization model for load prediction. In [10] an aggregated load profile prediction for a community is calculated using a two-step mechanism. The first step uses LSTM for predicting weather conditions and the second one uses ANN to associate the weather prediction to the community power demand. Kong et al. (2017) [11] proposed a LSTMbased residential load forecasting framework taking into consideration appliance-level consumption. Other works correlate extra features in the prediction, such as temperature, day of the week, humidity, among others, exploiting deep neural networks [12]. Nevertheless, no one of these approaches tackles the problem of a continuous load prediction with incremental learning as input for optimal control.

The following chapters will present the methodology used for selecting the most appropriate algorithm, the experimental setup, the results and evaluation of them and conclusions.

\section{Methodology}

In this section, we will first give a brief introduction to recurrent neural networks (RNN) based on long short-term memory (LSTM) units and discuss the motivation for the choice of this model. Then, we will focus on potential optimizations for RNN and the actual domain-specific implementation.

\section{A. Recurrent Neural Networks with LSTM units}

Although there is a broad landscape of prediction models [13], ranging from statistical approaches like the autoregressive-moving-average [14] up to common machine learning techniques as kernelized support vector machines [15], most models tend to struggle when it comes to efficient onlinelearning. For some of them, the reason is the lack of flexibility regarding complex non-linear functions including long-term dependencies. Others are not suitable, since they do not support an incremental processing of incoming data.

While a training via stateful RNNs allows to inherently solve the latter problem, due to LSTM units they are also capable of capturing different temporal correlations in the data. From a high-level view, RNNs can be thought of as a concatenation of multi-layer perceptrons with equivalent processing units, in order to process sequences using a nonlinear function $\mathrm{f}$ :

$$
X \in \mathbb{R}^{24 \times 1}, y^{\prime} \in \mathbb{R}^{24 \times 1}: \quad f(X) \rightarrow y^{\prime}
$$

For a daily prediction, in equation (1) we already defined the number of time steps of input and ouput to be 24, assuming hourly measured data. Note that for other scenarios those dimensions can vary, i.e. for a prediction of multidimensional data with a different resolution. Since in general vanilla RNNs have difficulties learning long-term-dependencies, the usage of LSTM units can help to adapt to different temporal correlations. A detailed introduction to LSTMs can be found in [16].

\section{B. Optimizations}

Refining the efficiency of neural networks can become a difficult task, which is primarily dependent on the underlying data. In the following, we will shortly outline two popular regularization approaches for the optimization of RNNs, which are also considered in the upcoming evaluation section.

Independently from tuning model-specific hyperparameters, regularization constitutes a potential improvement with respect to a tradeoff between reducing the bias while simultaneously reducing the variance [17]. Here, in addition to a minimization of the original cost function, a penalty-term is added in order to reduce an overfitting of the model according to the training data. This approach can be used since the behavior of new incoming data might not be perfectly represented by historical data. However, literature has shown that especially for RNNs, regularization might decrease the exhibition of long-term memory traces [18].

An alternative to this regard is dropout [19], where during the learning phase, nodes of the network might be randomly excluded from the cost function or not, depending on a predefined parameter. This variation of the cost function can therefore lead to a lower variance, and thus to a less sensitive prediction model.

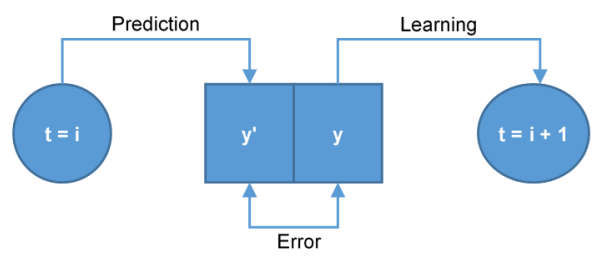

Figure 1. Incremental prediction/learning proces

\section{RNN (LSTM) for Energy Load Prediction}

Even though the usage of RNNs for regression is not new on its own, to the best of our knowledge, so far there is no previous work related to the problem of exploiting LSTMs for incremental learning in the context of continuous energy load prediction as input for optimization problems. Furthermore, the upcoming model is additionally used as an input for a subsequent optimization framework as presented in [4].

Given a data stream of time-annotated energy load observations providing measurements at a nearly constant rate, the approach in this study uses a stateful RNN with LSTM units for incremental learning and prediction. Incremental refers to the fact that at each time-interval, the RNN predicts new values $y^{\prime}$ for the upcoming time steps, while it also adapts itself based on the real observed values $y$. An illustration of this process is provided in Fig. 1, where the predictions at each time step $i$ are compared against the ground truth and used for a subsequent 
retraining. As the RNN learns and predicts using intervals of observations to inherently capture a specific behavior, for both steps the input $X$ consists of the last $p$ (in our case 24) observations. For an initial prediction, where the number of observations is smaller than $p$, the RNN can be substituted by a baseline model, which uses the last observed value as a prediction of $y^{\prime}$.

The goal at each incremental learning step is consequently to minimize the error $\left|y^{\prime}-y\right|$ according to every subsequent prediction.

Beside the task of efficient online learning, another challenge is to match the requirement of deploying the LSTM model into the optimization framework, where it serves as input of an optimization model. As the framework allows the user to choose the optimization horizon and horizon time resolution, the predictions using the LSTM model should be able to cope with this flexibility.

\section{EXPERIMENTAL SETUP}

The experimental setup, i.e., the data used, methods employed, tools utilized in the implementation and procedures followed for this study are explained as follows:

\section{A. Data}

The proposed model was tested on the energy consumption data (in KiloWatt) obtained from OpenEI [20]. This dataset contains information about energy consumption in different cities of the United States of America. The data is available for a period of one year in a resolution of one hour (Fig. 2). The proposed model was also tested on energy consumption data obtained in real-time from smart meters deployed at residential houses in Denmark in the context of the Storage4Grid (S4G) project (Fig. 3). The data is available for a period of three months with a secondly time resolution.

\section{B. Implementation}

According to Section II.C, we used a Long-Short-TermMemory (LSTM) based Recurrent Neural Network (RNN) to perform the task of load forecasting with incremental learning. In particular, we used stateful LSTMs in Keras, because they allow maintaining cell state among various training batches. This generally is required when there is periodicity in the data, as in the case of household consumption. Here, consumption patterns vary according to the time of day and time of year.

The LSTM model keeps training continuously as new realtime data arises in order to adjust its parameters. Consequently, the predictions are calculated using the updated LSTM model. This characteristic is considered and fits with the implementation of the incremental learning LSTM into the optimization platform, which uses real-time and historical data for the incremental learning of the model. In this case, real-time data from the smart meter is stored into the non-volatile memory periodically and used for the further training of the model.

For the dataset from Denmark, the samples within a minute were averaged, in order to change the time resolution to minutes. The objective is to comply with the minimal optimal control frequency set by the optimization framework which is
1 min. Afterwards, the data was normalized using quantile normalization. Although the normalization has no further impact in the current scenario, it is still considered in this approach to potentially speed up the gradient descent algorithm, inherently applied by the RNN, when working with multivariate data. Due to the requirements, established by the real-time system in the optimization platform, the data could not be normalized using the maximal value of the inputs. In a real-time system, the maximum consumption value is not known beforehand, as it depends on several factors such as number and type of electrical devices, as well as user behavior and preferences. For this reason, a manually selected maximal value was used for the normalization.

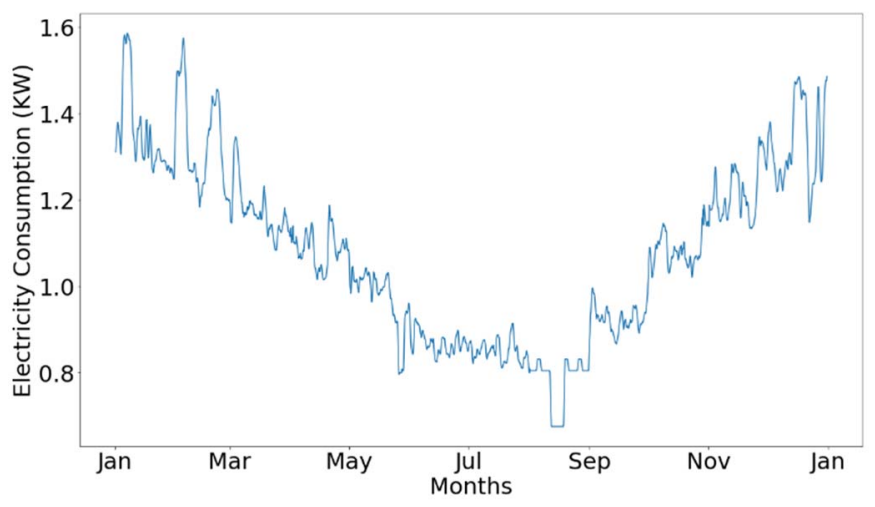

Figure 2. Raw data from House 1 of OpenEI dataset in the USA

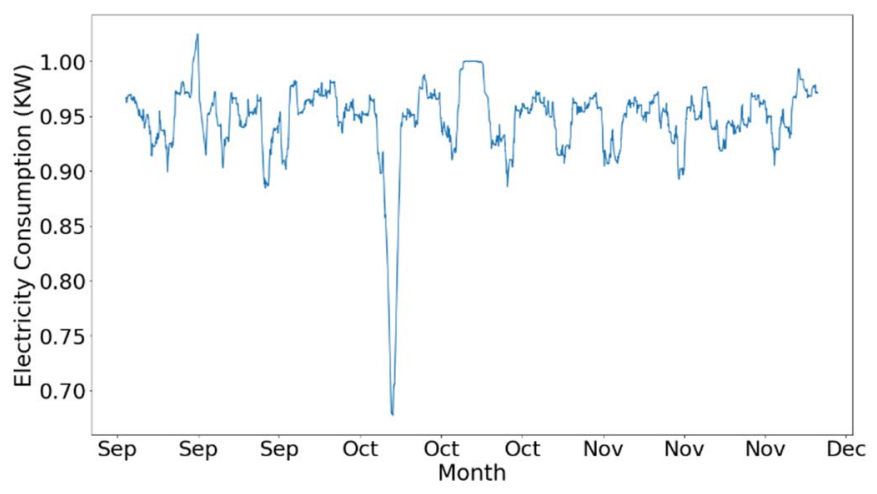

Figure 3. Raw data from House 1 of Denmark dataset

We incrementally train the model with an input of 24 data points (equals 24 time steps) for 10 epochs and predict the values for the next day. The input is updated using a sliding window of size 1. Independent of the offset regarding training and prediction, both processes are done simultaneously.

The programming language used for implementation of the tasks in this study is Python due to the availability of flexible libraries and portability across platforms. We used Keras framework with TensorFlow [21] as backend for implementing LSTM. To implement the statistical model ARIMA, a python module StatsModels was used. The computations were done using a computer with Intel(R) Core(TM) i7-6600U CPU @ $2.60 \mathrm{GHz} 2.80 \mathrm{GHz}$. 


\section{Procedures}

The first step in our work was the comparison of the LSTM algorithm with the statistical ARIMA algorithm. In this case, we used one USA dataset from OpenEI and analyzed both algorithms running on it. We used a batch-wise approach using the twelve months as training data but without the last day, which was used for the prediction.

In the second step, we optimized the LSTM algorithm for our application, as it is required to provide continuous input for optimization models. As we explained in Section III.B, this behavior was simulated with the USA dataset from OpenEI. In order to optimize the RNN, we evaluated different parameters like the number of stacked layers and nodes per layer as well as additional regularization techniques including Lasso-, Ridge regularization (L1- L2 regularization), Dropout and a combination of them both.

Finally, we used the optimal LSTM configuration and evaluated it with nine more houses of USA dataset from OpenEI and the five-household dataset from Denmark. In the same way, we simulated continuous operation as explained in Section III.B.

\section{RESUlTS AND EVALUATION}

The comparison between the statistical ARIMA algorithm and the LSTM RNN using one USA dataset of openEI, showed that the LSTM model presented a better behavior while predicting the load for the next day. The results presented in Fig. 4 show that the results using the LSTM algorithm fits the original data better than the results using ARIMA. Since in addition ARIMA cannot be trained incrementally - for each retraining, the whole data is necessary - in connection with the given requirements, we did not investigate more promising variants of ARMIA as for instance SARIMA. Therefore, in the following only the LSTM was investigated in more detail.

For the further evaluation steps, we chose the common mean-absolute error deviation (MAE) and root-mean-square error (RMSE) as common evaluation metrics. Therefore, the results are presented based on the obtained MAE and RMSE values over time. Additionally, we chose the number of time steps (look-back features) to be 24 , i.e. one full day. This was done to implicitly learn a daily behavior from the openEI dataset. We continued using this value for the Denmark dataset, even though the original time resolution is higher. Since the training is performed incrementally for single batches, the batch size was accordingly set to 1 .

In addition to those default settings, we present a comprehensive evaluation of our chosen optimization parameters for the LSTM model using one USA dataset of openEI (House 1). As such, we run experiments for different combinations of scenarios and obtained their results in form of MAE and RMSE (Table 1).

In order to take a decision on how many hidden nodes to use, we conducted an experiment using varied number of hidden nodes. The prediction performance steadily improved when changing the number of hidden nodes from 20 until 120. We used 100 hidden nodes for subsequent experiments, because it showed the least error for openEI House 1 (Fig. 5).
Due to the superior performance in combination with regularization techniques, we decided to continue using stacked layers, instead of a single layered architecture

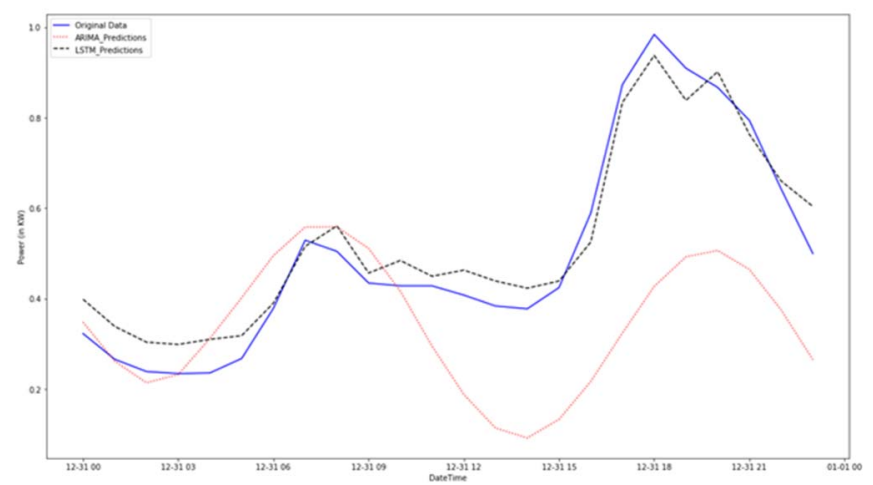

Figure 4. Preliminary experiment to compare ARIMA and LSTM

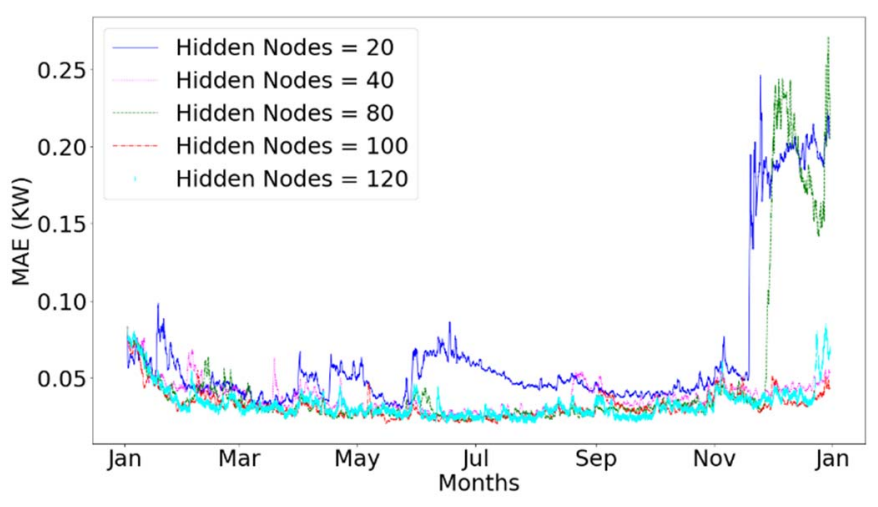

Figure 5. Prediction error with varied number of hidden nodes

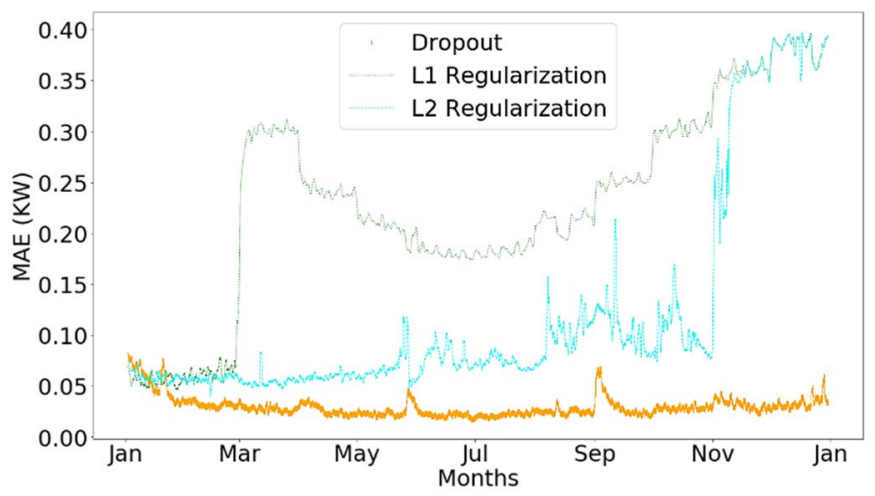

Figure 6. Prediction error with $\mathrm{L} 1$ and $\mathrm{L} 2$ regularization and dropout

We conducted experiments using dropout, L1 and L2 regularization and a combination of both (Fig. 6). Dropout was chosen to reduce overfitting by randomly dropping hidden units during training from the neural network and preventing units from co-adapting. L1 and L2 regularization were used to decrease the variance for upcoming predictions (at the cost of an increased bias) [18]. As shown in Table 1, the prediction performance of the model that uses stacked LSTM in combination with dropout of 0.3 presented the lowest MAE and RMSE. Moreover, the training time of $460 \pm 34.25 \mathrm{~ms}$ and the 
prediction time of $4.11 \pm 0.37 \mathrm{~ms}$ analyzed during the whole year using the last LSTM configuration allows the use of the LSTM algorithm for continuous load prediction with incremental learning in the platform specified in Section II.B. Fig. 8 and Fig. 9 show the training and prediction time using the best performing model over one year period.

Additionally, we compared the best performing model with the baseline model, as shown in Fig. 7, in order to test the prediction quality of LSTM compared with a basic prediction model as the baseline model is. The MAE and RMSE values over the time were significantly lower and steady for LSTM as for the baseline model.

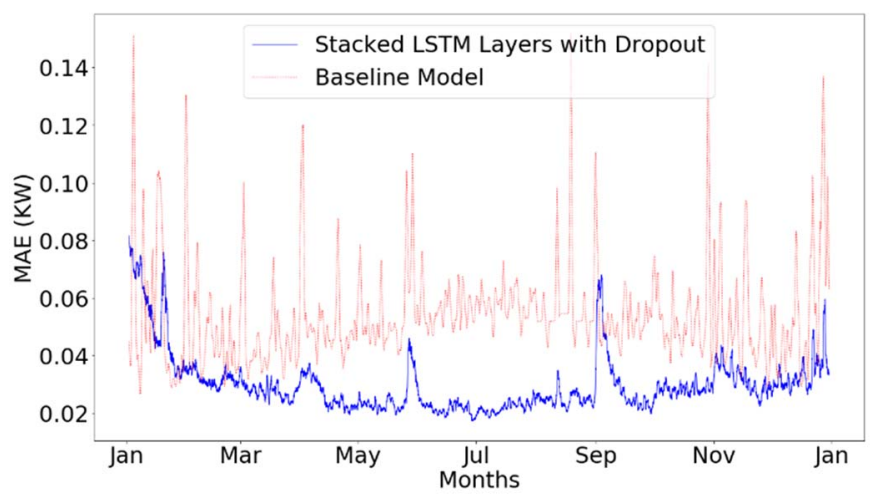

Figure 7. Prediction error of Baseline vs Stacked LSTM Models

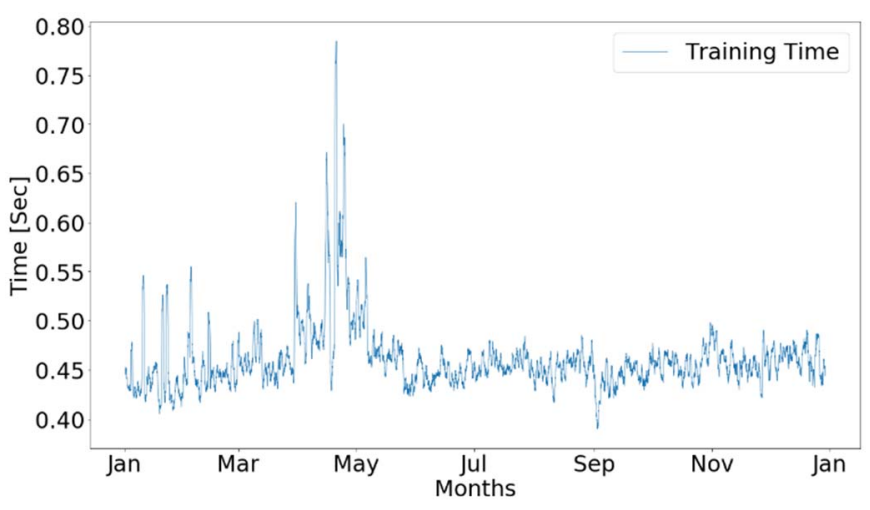

Figure 8. Best performing model's training time

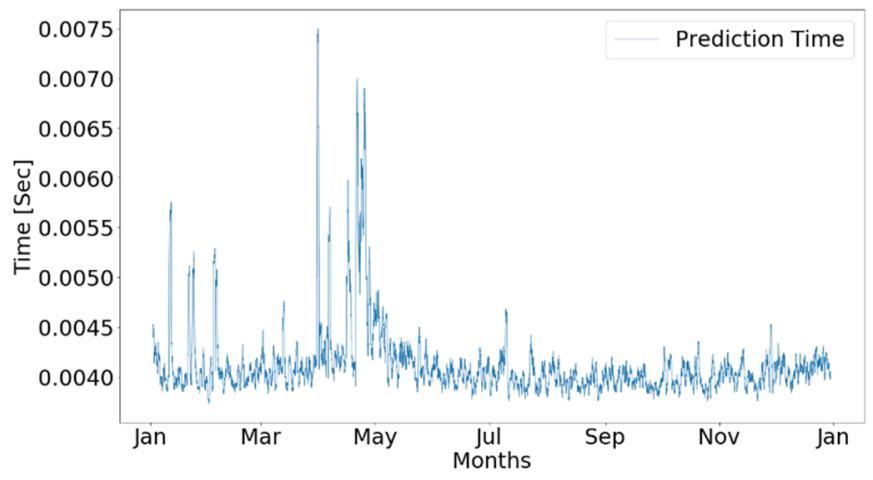

Figure 9. Best performing model's prediction time

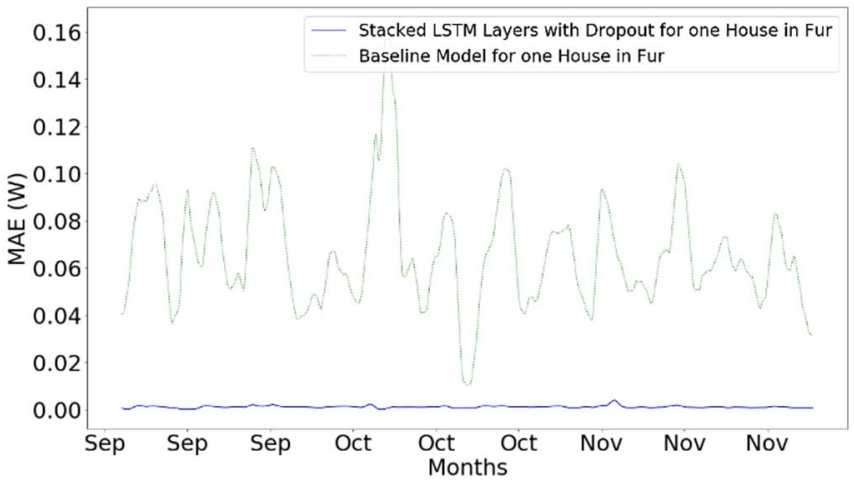

Figure 10. Baseline vs chosen model error in one household of Denmark

TABLE I. MODEL WITH VARIOUS SCENARIOS FOR THE NEXT 24 HOURS FOR HOUSE 1 OF THE OPENEI DATASET

\begin{tabular}{c|c|c|c|c} 
HLayer & $\begin{array}{c}\text { Nod } \\
\mathbf{e}\end{array}$ & Regularization & $\begin{array}{c}\text { MAE } \\
(\mathbf{k W})\end{array}$ & RMSE \\
\hline 1 & 20 & - & 0,0651 & 0.0845 \\
\hline 1 & 40 & - & 0,0377 & 0.0400 \\
\hline 1 & 80 & - & 0,0471 & 0.0705 \\
\hline 1 & 100 & - & 0,0321 & 0.0341 \\
\hline 1 & 120 & - & 0,0355 & 0.0361 \\
\hline 2 & 100 & - & 0,0576 & 0.0637 \\
\hline 2 & 100 & Dropout(0.1) & 0,0345 & 0.0431 \\
\hline 2 & 100 & Dropout $(0.2)$ & 0,0311 & 0.0339 \\
\hline 2 & 100 & Dropout $(0.3)$ & 0,0299 & 0.0330 \\
\hline 2 & 100 & Dropout(0.4) & 0,0320 & 0.0349 \\
\hline 2 & 100 & Dropout(0.5) & 0,1126 & 0.1741 \\
\hline 2 & 100 & Dropout(0.6) & 0,0320 & 0.0359 \\
\hline 2 & 100 & Dropout(0.7) & 0,0392 & 0.0945 \\
\hline 2 & 100 & L1 (Alpha $=0.01)$ & 0,2291 & 0.2488 \\
\hline 2 & 100 & L2 (Alpha $=0.01)$ & 0,1217 & 0.1642 \\
\hline 2 & 100 & Dropout(0.3), L1 (Alpha $=0.01)$ & 0,2098 & 0.2343 \\
\hline 2 & 100 & Dropout(0.3), L2 (Alpha $=0.01)$ & 0,1945 & 0.2227
\end{tabular}

TABLE II. MODEL WITH VARIOUS SCENARIOS FOR THE NEXT 24-HOURS

\begin{tabular}{c|c|c|c|c|c} 
Dataset & HLayer & Nodes & Regularization & MAE & RMSE \\
\hline openEI - 1 & 2 & 100 & Dropout $(0.3)$ & 0,0299 & 0.0330 \\
\hline openEI - 2 & 2 & 100 & Dropout $(0.3)$ & 0,0334 & 0.0371 \\
\hline openEI - 3 & 2 & 100 & Dropout $(0.3)$ & 0,0701 & 0.1062 \\
\hline openEI - 4 & 2 & 100 & Dropout $(0.3)$ & 0,0321 & 0.0353 \\
\hline openEI - 5 & 2 & 100 & Dropout $(0.3)$ & 0,0314 & 0.0346 \\
\hline openEI - 6 & 2 & 100 & Dropout $(0.3)$ & 0,0294 & 0.0319 \\
\hline openEI - 7 & 2 & 100 & Dropout $(0.3)$ & 0,0331 & 0.0359 \\
\hline openEI - 8 & 2 & 100 & Dropout $(0.3)$ & 0,0293 & 0.0322 \\
\hline openEI - 9 & 2 & 100 & Dropout $(0.3)$ & 0,0314 & 0.0342 \\
\hline openEI - & 2 & 100 & Dropout $(0.3)$ & 0,0333 & 0.0369 \\
10 & & & & & \\
\hline Denmark-1 & 2 & 100 & Dropout $(0.3)$ & 0,0079 & 0.0091 \\
\hline Denmark-2 & 2 & 100 & Dropout $(0.3)$ & 0,0052 & 0.0066 \\
\hline Denmark-3 & 2 & 100 & Dropout $(0.3)$ & 0,0046 & 0.0053 \\
\hline Denmark-4 & 2 & 100 & Dropout $(0.3)$ & 0,0075 & 0.0079 \\
\hline Denmark-5 & 2 & 100 & Dropout $(0.3)$ & 0,0044 & 0.0048
\end{tabular}

Due to its performance, we continue analyzing the stacked LSTM in combination with dropout of 0.3 with nine further houses of the openEI dataset and with five houses of the Denmark dataset. The results are presented in Table 2 and show a low MAE for all houses with a maximal value of $0.070 \mathrm{~kW}$. The low MAE confirms that the suitability of the best performing LSTM model is not limited to the first openEI 
dataset but can be applied to all load profile datasets, even though they present different behavior.

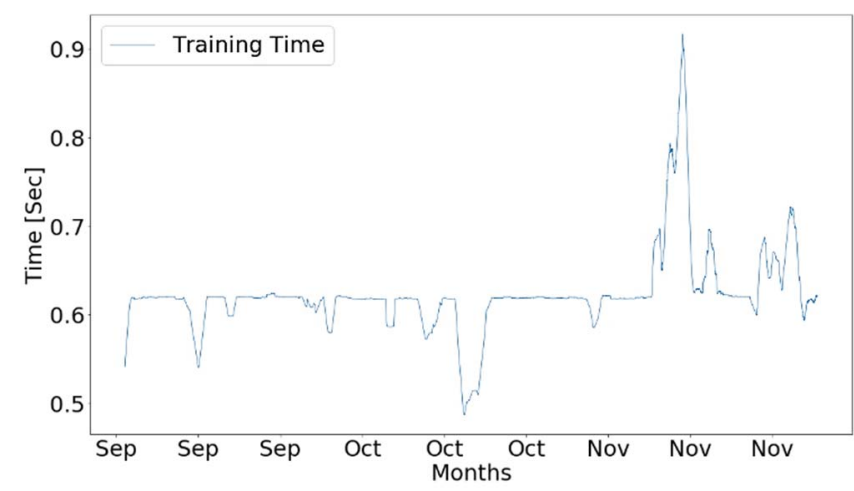

Figure 11. Training time in one household of Denmark

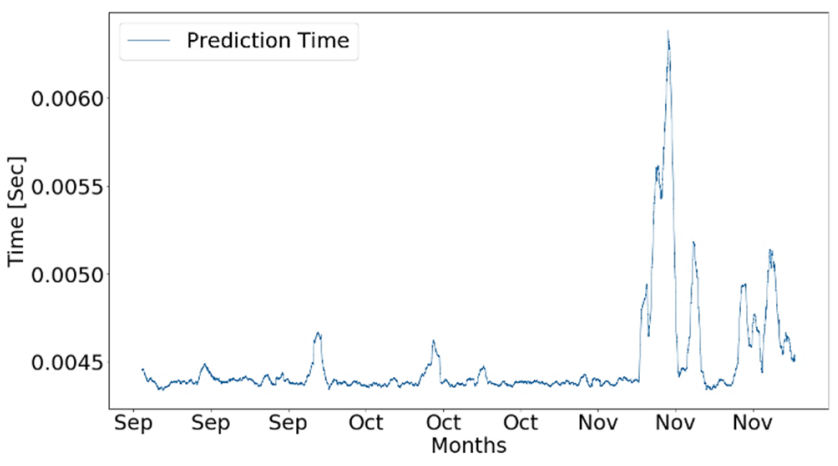

Figure 12. Prediction time in one household of Denmark

Fig. 10 shows a comparison between the baseline model and the best performing LSTM model for one household in Denmark. Similar to the case of openEI dataset, the training time $(622 \pm 46.8 \mathrm{~ms})$ and prediction time $(4.49 \pm 0.27 \mathrm{~ms})$ with the dataset of Denmark remained low (Fig. 11 and Fig. 12). This confirms the applicability of the model for continuous load prediction in households in different locations.

\section{CONCLUSIONS AND FUTURE WORKS}

This work sets out to develop a model and procedure that utilizes smart meter data to predict the energy consumption of a household. After conducting a series of experiments, the study found an optimal LSTM model that enables a continuous load prediction with incremental learning, which fits optimally the behavior of the real data with MAE below 0.1. The future work of this study is to perform further experiments at different levels of time granularity and with longer real household data. Moreover, we will include the prediction algorithm in our optimization framework, in order to achieve continuous optimal control of complex systems that helps users make better use of the consumed energy. To achieve it, the LSTM will be evaluated in different hardware platforms. We will also include electric vehicle load in the prediction as one possibility for optimization.

\section{ACKNOWLEDGEMENTS}

This project has received funding from the European Union's Horizon 2020 research and innovation program under grant agreement No 731155. The sole responsibility of this publication lies with the author. The European Union is not responsible for any use that may be made of the information contained therein.

\section{REFERENCES}

[1] Bundesministerium für Umwelt, Naturschutz, and Bau und Reaktorsicherheit, "Klimaschutzplan 2050 - Klimaschutzpolitische Grundsätze und Ziele der Bundesregierung," Klimaschutzplan 2050, pp. 1-96, 2016.

[2] B. K. Sovacool, "Valuing the greenhouse gas emissions from nuclear power: A critical survey," Energy Policy, vol. 36, no. 8, pp. 2940-2953, 2008.

[3] AG Energiebilanzen e.V., "Auswertungstabellen zur Energiebilanz Deutschland. 1990 bis 2017 (Stand Juli 2018)," no. 030, 2018.

[4] A. C. G. Aragón, E. Gümrükcü, "Optimization Framework for shortterm control of Energy Storage Systems," 2018 IEEE PES Innov. Smart Grid Technol. Conf. Eur., 2018.

[5] K. Chen and C. Wang, "A hybrid SARIMA and support vector machines in forecasting the production values of the machinery industry in Taiwan," vol. 32, pp. 254-264, 2007.

[6] "A New Method of Load Forecasting Based on Generalized Autoregressive Conditional Heteroscedasticity Model-- 《Automation of Electric Power Systems》2007年15期.” [Online]. Available: http://en.cnki.com.cn/Article en/CJFDTOTAL-DLXT200715012.htm. [Accessed: 15-Nov-2018].

[7] X. Cao and S. Dong, "A Data-driven Hybrid Optimization Model for Short- term Residential Load Forecasting," 2015.

[8] S. Skolthanarat, U. Lewlomphaisarl, and K. Tungpumolrut, "Short-term Load Forecasting Algorithm and Optimization in Smart Grid Operations and Planning," IEEE Conf. Technol. Sustain., pp. 165-171, 2014.

[9] N. Tang and D. J. Zhang, "Application of a load forecasting model based on improved grey neural network in the smart grid," Energy Procedia, vol. 12, pp. 180-184, 2011.

[10] M. Cenek, R. Haro, B. Sayers, and J. Peng, "Climate Change and Power Security: Power Load Prediction for Rural Electrical Microgrids Using Long Short Term Memory and Artificial Neural Networks," Appl. Sci., vol. 8, no. 5, p. 749, 2018.

[11] W. Kong et al., "Short-Term Residential Load Forecasting based on LSTM Recurrent Neural Network," vol. 3053, no. c, pp. 1-11, 2017.

[12] W. He, "Load Forecasting via Deep Neural Networks," Procedia Comput. Sci., vol. 122, pp. 308-314, 2017.

[13] K. P. Murphy, Machine Learning: A Probabilistic Perspective. The MIT Press, 2012.

[14] G. E. P. Box, G. M. Jenkins, G. C. Reinsel, and G. M. Ljung, Time series analysis : forecasting and control. .

[15] B. E. Boser, I. Guyon, and V. V. A, "Training Algorithm for Optimal Margin Classi Ers," Proc. Fifth Annu. Work. Comput. Learn. Theory, vol. pages, pp. 144-152.

[16] S. Hochreiter and J. Schmidhuber, "Long Short-Term Memory," Neural Comput., vol. 9, no. 8, pp. 1735-1780, Nov. 1997

[17] H. Zou and T. Hastie, "Regularization and variable selection via the elastic net,” J. R. Stat. Soc. Ser. B Stat. Methodol., vol. 67, no. 2, pp. 301-320, 2005.

[18] M. T. and B. Y. Pascanu R., "On the difficulty of training of recurrent neural networks," Int. Multi-Conference Syst. Signals Devices, SSD’11 - Summ. Proc., 2011.

[19] T. Wunderling, B. Golla, P. Poudel, C. Arens, M. Friebe, and C. Hansen, "Dropout: A Simple Way to Prevent Neural Networks from Overfitting," vol. 15, p. 1013317, 2017.

[20] "Index of /datasets/files/961/pub." [Online]. Available: https://openei.org/datasets/files/961/pub/. [Accessed: 26-Nov-2018].

[21] H. Wermser, A. Rettinger, and V. Tresp, "Modeling and learning context-aware recommendation scenarios using tensor decomposition," Proc. - 2011 Int. Conf. Adv. Soc. Networks Anal. Mining, ASONAM 2011, pp. 137-144, 2011 